\title{
Current Perspectives on Inflammation in Cardiovascular Disease; from Biomarker to Therapy
}

\author{
Saskia C. A. de Jager ${ }^{1}$ • Joost P. G. Sluijter ${ }^{1}$ \\ Published online: 30 August 2020 \\ (C) Springer Science+Business Media, LLC, part of Springer Nature 2020
}

Inflammation has been a prominent topic of research in cardiovascular disease over the last decades. This special issue $(S I)$ entitled "Current perspectives on inflammation in cardiovascular disease; from biomarker to therapy" contains 9 selected reviews that feature the different aspects of the inflammatory response in cardiovascular disease. Overall, this SI highlights the future directions on the use of immunomodulatory therapies for cardiovascular disease.

This $S I$ starts with a focus on vascular disease, where Inia and O'Brien provide an overview on the atheroprotective role of Heat Shock Protein-27 that might be a promising therapeutic target. Furthermore, it is suggested that the HSP27estrogen association may be important in the protection against atherosclerosis development in women [1]. The paper by Baganha and colleagues focuses on adverse vein graft remodeling. Vein grafts have to adjust to arterial pressure and it is well established that the inflammatory response is crucial for physiological vein graft adaptation and long-term patency, but can also lead to vein graft failure. Here, pathophysiological mechanisms are described, highlighting the role for immune cells underlying vein graft failure and providing insight in novel therapeutics to improve vein graft patency [2].

The promising results of the CANTOS trial has (re)inspired many researchers in the field to bring back more antiinflammatory approaches into the cardiovascular clinical arena. Bosmans and colleagues discuss the involvement of the CD40/CD40L dyad in atherosclerosis, which has recently resulted in the development of novel therapeutics [3]. Much

Saskia C. A. de Jager

S.C.A.deJager@umcutrecht.nl

1 Laboratory for Experimental Cardiology and Center for Regenerative Medicine, University Medical Center Utrecht, University Utrecht, Utrecht, The Netherlands time, effort, and energy is currently being put into therapeutic targeting of the nucleotide-binding oligomerization domainlike receptor family pyrin domain containing 3 (NLRP3) inflammasomes, which contributes to the release of interleukin- $1 \beta$. Silvis and colleagues summarize the role of the NLRP3 inflammasome and provide a perspective on the current therapeutic approaches targeting the NLRP3 inflammasome for cardiovascular disease [4].

Chronic recruitment of inflammatory cells to the myocardium contributes to adverse remodeling and the onset of heart failure. Chen and Frangogiannis describe the current knowledge on the role of the chemokine family in injury, repair, and remodeling upon cardiac ischemia [5]. Furthermore, they discuss both the potential as well as possible drawbacks for targeting the chemokine family. Consequential to myocardial ischemia reperfusion injury, heart failure often develops for which diagnosis mostly relies on circulating biomarkers and loss of cardiac function. Meier and colleagues discuss that generic biomarkers often lack sensitivity for many patients and they propose that circulating white blood cells reflect molecular changes in the heart and are the source of next-generation molecular biomarkers [6].

Hand in hand with the urgent need for novel biomarkers to identify early heart failure patients comes the need for (curative) therapies. Particularly in heart failure with preserved ejection fraction (HFpEF), the systemic pro-inflammatory state, consequential to underlying comorbidities, is believed to contribute to a decline in cardiac function. Currently available heart failure treatments mostly failed to demonstrate a clear benefit in HFpEF patients. Therefore, Kessler and colleagues point out that therapies underlying comorbidities and systemic inflammation in early HFpEF may provide better opportunities for treating these patients. In their review in this SI, they provide an overview of the current state and future perspectives of immunomodulatory therapies for HFpEF [7]. 
The characteristic inflammatory reaction in myocarditis can, when progressed into a state of chronic inflammation, result in outward remodeling and loss of cardiac function, which is clinically referred to as inflammatory cardiomyopathy (Infl-CM). Elsanhoury and colleagues describe that Infl-CM remains an unmet medical need [8]. Several therapeutic targets, revealed by basic research, still lack translation to the patient. The current immunosuppressive strategies are mainly based on the use of harsh immunosuppressive agents and are often tested in small-scale, single-center cohort studies, stressing the need for large-scale randomized placebo controlled clinical studies to designate safe and effective treatments for Infl-CM.

Regeneration of cardiac tissue holds great potential for the prevention of adverse remodeling. Particularly, the transplantation of cardiac progenitor or stem cells is believed to improve cardiac regenerative capacity; however, the field has been disappointed on several occasions as the transplantation of these cells often fail to improve cardiac function. An important contributor that influences long-term integration may be the recipient's immune defense system. In this review, Demkes and colleagues assess the immunological barriers regarding non-autologous cell transplantation and the importance of immune modulation with immunosuppressive drugs. In addition, they stress the importance of proper immunosuppression in large animal models and provide clear recommendations for immunosuppressive regimens in preclinical cardiac cell-replacement studies [9].

Overall, the collection of papers in this SI not only provides a clear overview on the importance of inflammation and the potential promise in targeting the inflammatory component in cardiovascular disease but it also provides critical mass on the expected hurdles that have to be overcome before we can actually clinically apply these promising novel therapies to those patients that benefit the most.

\section{References}

1. Inia, J., \& O'Brien, E. (2020). Role of Heat Shock Protein 27 in modulating atherosclerotic inflammation. Journal of Cardiovascular Translational Research. https://doi.org/10.1007/ s12265-020-10000-z.

2. Baganha, F., de Jong, A., Jukema, J. W., Quax, P. H. A., \& de Vries, M. R. (2020). The role of immunomodulation in vein graft remodeling and failure. Journal of Cardiovascular Translational Research. https://doi.org/10.1007/s12265-020-10001-y.

3. Bosmans, L. A., Bosch, L., Kusters, P. J. H., Lutgens, E., \& Seijkens, T. T. P. (2020). The CD40-CD40L dyad as immunotherapeutic target in cardiovascular disease. Journal of Cardiovascular Translational Research. https://doi.org/10.1007/s12265-02009994-3.

4. Silvis, M., Demkes, E. J., Fiolet, A. T. L., Dekker, M., Bosch, L., van Hout, G. P. J., et al. (2020). Immunomodulation of the NLRP3 inflammasome in atherosclerosis, coronary artery disease and acute myocardial infarction. Journal of Cardiovascular Translational Research. https://doi.org/10.1007/s12265-020-10049-w.

5. Chen, B., \& Frangogiannis, N. G. (2020). Chemokines in myocardial infarction. Journal of Cardiovascular Translational Research. https://doi.org/10.1007/s12265-020-10006-7.

6. Meier, S., Henkens, M., Heymans, S., \& Robinson, E. L. (2020). Unlocking the value of white blood cells for heart failure diagnosis. Journal of Cardiovascular Translational Research. https://doi.org/ 10.1007/s12265-020-10007-6.

7. Kessler, E. L., Oerlemans, M., van den Hoogen, P., Yap, C., Sluijter, J. P. G., \& de Jager, S. C. A. (2020). Immunomodulation in heart failure with preserved ejection Fraction: Current State and Future Perspectives. Journal of Cardiovascular Translational Research. https://doi.org/10.1007/s12265-020-10026-3.

8. Elsanhoury, A., Tschope, C., \& Van Linthout, S. (2020). A toolbox of potential immune-related therapies for inflammatory cardiomyopathy. Journal of Cardiovascular Translational Research. https://doi. org/10.1007/s12265-020-10025-4.

9. Demkes, E. J., Rijken, S., Szymanski, M. K., Hoefer, I. E., Sluijter, J. P. G., \& de Jager, S. C. A. (2020). Requirements for proper immunosuppressive regimens to limit translational failure of cardiac cell therapy in preclinical large animal models. Journal of Cardiovascular Translational Research. https://doi.org/10.1007/ s12265-020-10035-2.

Publisher's Note Springer Nature remains neutral with regard to jurisdictional claims in published maps and institutional affiliations. 